\title{
Outcome of hospital discharge on postoperative Day 1 following uncomplicated tethered spinal cord release
}

\author{
Seerat Poonia, BA, ${ }^{1}$ Sarah Graber, BA, ${ }^{2}$ C. Corbett Wilkinson, MD, ${ }^{2}$ Brent R. O'neill, MD, ${ }^{2}$ \\ Michael H. Handler, MD, ${ }^{2}$ and Todd C. Hankinson, MD, MBA ${ }^{2,3}$ \\ ${ }^{1}$ University of Colorado School of Medicine; ${ }^{2}$ Pediatric Neurosurgery, Children's Hospital Colorado, University of Colorado \\ Anschutz Medical Campus; and ${ }^{3}$ Adult and Child Center for Health Outcomes Research and Delivery Science, University of \\ Colorado Anschutz Medical Campus, Aurora, Colorado
}

\begin{abstract}
OBJECTIVE Postoperative management following the release of simple spinal cord-tethering lesions is highly variable. As a quality improvement initiative, the authors aimed to determine whether an institutional protocol of discharging patients on postoperative day (POD) 1 was associated with a higher rate of postoperative CSF leaks than the prior protocol of discharge on POD 2.

METHODS This was a single-center retrospective review of all children who underwent release of a spinal cord-tethering lesion that was not associated with a substantial fascial or dural defect (i.e., simple spinal cord detethering) during 2 epochs: prior to and following the institution of a protocol for discharge on POD 1. Outcomes included the need for and timing of nonroutine care of the surgical site, including return to the operating room, wound suturing, and nonsurgical evaluation and management.
\end{abstract}

RESULTS Of 169 patients identified, none presented with CSF-related complications prior to discharge. In the preintervention group ( $n=113$ ), the postoperative CSF leak rate was $4.4 \%(5 / 113)$. The mean length of stay was 2.3 days. In the postintervention group, the postoperative CSF leak rate was 1.9\% (1/53) in the patients with postdischarge follow-up. The mean length of stay in that group was 1.3 days.

CONCLUSIONS At a single academic children's hospital, a protocol of discharging patients on POD 1 following uncomplicated release of a simple spinal cord-tethering lesion was not associated with an increased rate of postoperative CSF leaks, relative to the previous protocol. The rates identified are consistent with the existing literature. The authors' practice has changed to discharge on POD 1 in most cases.

http://thejns.org/doi/abs/10.3171/2015.10.PEDS15318

KEY WORDS tethered spinal cord; occult spinal dysraphism; postoperative management; cerebrospinal fluid leak; pseudomeningocele; spine

$\mathrm{O}$ CCULT spinal dysraphism (OSD) is a commonly encountered entity in pediatric neurosurgical practice and includes a number of pathological entities that are associated with functional spinal cord tethering. The majority of these congenital lesions are not associated with substantial dural or fascial defects and can be addressed through a single-level laminotomy or laminectomy and small durotomy. ${ }^{1,8}$ Common lesions in this category include fibrolipoma of the filum terminale, thickening of the filum terminale, and dermal sinus tract without an intraspinal dermoid cyst.

Although many reports detail neurological and urological outcomes following surgical intervention for OSD, ${ }^{3-6,9}$ there is less discussion in the literature regarding periop- erative management protocols for variants of OSD that are not associated with substantial tissue defects. ${ }^{1,7}$ Because many patients have minimal or no symptoms at the time of operative detethering, the importance of complication avoidance in this population is paramount. There is little debate that a period of recumbency following tethered cord release probably lowers the risk of a CSF leak, but the optimal duration of this period is not well defined. ${ }^{1,2,7}$ Chern and colleagues demonstrated, across 3 institutions, that the duration for which a patient was kept flat in the postoperative setting did not correlate with the likelihood of a CSF leak or pseudomeningocele. ${ }^{1}$ Recently, Ogiwara and colleagues ${ }^{7}$ reported no CSF leaks in a large population of patients who were kept flat for 72 hours. 
Before December 2013, our institutional practice was to maintain patients in a horizontal position for 24 hours after surgery, followed by a further 24 hours of inpatient observation to assess the individual for evidence of a pseudomeningocele or CSF leak. In the absence of concerning findings, patients were discharged home on postoperative day (POD) 2. We observed very few wound-related complications (e.g., a pseudomeningocele or CSF leak) during the period between the discontinuation of positioning restrictions and discharge the following day. We hypothesized that our practice of keeping patients in the hospital until POD 2 did not result in the more timely identification of CSF-related complications following simple tethered spinal cord release and overnight recumbent positioning. Beginning in December 2013, we modified our practice. We began to mobilize the patient on the morning of POD 1 , monitor the surgical site during POD 1, and discharge the patient on the afternoon of POD 1. This project was undertaken to assess whether this change in practice was associated with an increased rate of CSF-related complications in our population of children who underwent simple tethered spinal cord release.

\section{Methods \\ Project Design}

This study was approved as a quality improvement project to assess an initiative to change our institutional practice from discharging patients on POD 2 following an uncomplicated laminotomy or laminectomy for release of a simple tethered spinal cord to discharge on the afternoon of POD 1. We conducted a retrospective review of the records of children who underwent operative release of a tethered spinal cord at Children's Hospital Colorado between September 2010 and June 2015. The change in practice was made on November 1, 2013. The preintervention group underwent operative detethering between September 1, 2010, and October 31, 2013. The postintervention group underwent operative detethering between December 15, 2013, and June 30, 2015. Patients who had surgery between November 1 and December 14, 2013, were not included in the analysis, to allow transition to a consistent management pattern among the multiple members of the clinical service.

\section{Patient Population}

Using the electronic medical record, we identified all patients treated with Current Procedural Terminology (CPT) code 63200 (Laminectomy, with release of tethered spinal cord, lumbar). A "simple" tethering lesion was defined as one that was not associated with a dural or fascial defect that required tissue mobilization or grafting for closure (e.g., fibrolipoma of the filum terminale, thickened filum terminale, or dermal sinus without intraspinal mass). Patients who underwent detethering of a lesion associated with a significant dural defect (e.g., myelomeningocele, dorsal or transitional lipomyelomeningocele) or a multilevel durotomy (e.g., tumor, selective dorsal rhizotomy) were excluded from analysis. Data were collected by a professional research assistant and medical student, and reviewed by the senior author.
Data included the procedure performed; length of admission from the time of surgery; additional procedures performed under the same anesthetic as for tethered cord release; reasons for hospitalization beyond POD 2; reasons for nonoperative neurosurgical care beyond routine postoperative management; and additional neurosurgical procedures performed within 2 weeks of tethered cord release. Routine postoperative management included monitoring of the operative site while the patient remained in the hospital as well as an outpatient follow-up inspection of the site approximately 7 days after surgery. We did not undertake routine postoperative imaging.

The primary outcome was return to the operating room for repair of a CSF leak or pseudomeningocele. Additional outcomes included any need for nonroutine neurosurgical management within 2 weeks of spinal cord detethering. This time point was selected to allow careful analysis of all CSF-related wound complications that may have been identified during the routine period of postoperative hospitalization. Patient management decisions, including return to the operating room, were based on the judgment of the treating surgeon and were not based on a previously defined protocol.

\section{Results \\ Operative Technique and Pain Control}

The patient population was drawn from the practices of 4 board-certified or -eligible pediatric neurosurgeons. There was no formal protocol with regard to operative technique or closure. Therefore, the use of tissue closure adjuncts, such as DuraSeal, was not standardized. In all cases, primary dural and fascial closure was achieved or attempted. The superficial tissue layers were closed with absorbable suture, including a subcuticular layer and fastdrying skin adhesive. No dressing was placed on the skin for patients of any age. No barrier was used for nontoilet-trained patients. Given the short incisions required for these procedures, perioperative pain management was undertaken with a combination of ketorolac and oral acetaminophen. Additional nonsteroidal antiinflammatory medications and opioids (oral or intravenous) were used on an as-needed basis. Epidural catheters were not used.

\section{Patient Sample and Aggregate Data}

One hundred thirteen patients underwent simple tethered spinal cord release during the preintervention period and 56 did so during the postintervention period, for a total sample size of 169 patients (Table 1). There were 81 girls $(47.9 \%)$, and the median age at surgery was 1.8 years. The mean and median times to discharge were both 2.0 days from surgery. No patient in either group required a return to the operating room due to a CSF leak prior to discharge. Nine of the 169 patients (5.3\%) were lost to follow-up after discharge and were excluded from the analysis of incision management. Five patients (3.1\%) returned to the operating room for repair of a CSF leak or a large pseudomeningocele, on PODs $4,4,7,8$, and 8 . Twenty-eight patients $(17.5 \%)$ required admission longer than was planned based on the epoch during which surgery occurred, 1 of whom required surgery for repair of a CSF leak on POD 4. 
TABLE 1. Demographic data in 169 patients with spinal cord release

\begin{tabular}{lcccc}
\hline \multicolumn{1}{c}{ Characteristic } & Total, $\mathrm{n}=169$ & Preintervention, $\mathrm{n}=113$ & Postintervention, $\mathrm{n}=56^{\mathrm{p} \text { V Value }}$ \\
\hline Median age in yrs, range & $1.8,0.1-16.4$ & $1.6,0.1-14.8$ & $2.1,0.3-16.4$ & 0.74 \\
\hline No. female (\%) & $81(47.9)$ & $53(46.9)$ & $28(50.0)$ & 0.70 \\
\hline No. w/ diagnosis (\%) & & & & 0.26 \\
\hline Fatty filum terminale & $126(74.6)$ & $88(77.9)$ & $38(67.9)$ & \\
\hline Low-lying conus w/o fatty filum & $40(23.7)$ & $23(20.4)$ & $17(30.4)$ & \\
\hline Dermal sinus tract & $3(1.8)$ & $2(1.8)$ & $1(1.8)$ & \\
\hline
\end{tabular}

* Statistical tests used: age, nonparametric t-test (Wilcoxon); sex, chi square test; diagnosis, Fisher's exact test.

\section{Preintervention Group}

There were 53 girls (46.9\%), and the median age at surgery was 1.6 years. Eighty-eight patients (77.9\%) were treated for a fatty infiltrated filum terminale (with or without a low-lying conus medullaris); 23 (20.4\%) were treated for a low-lying conus medullaris in the absence of fat in the filum terminale; and $2(1.8 \%)$ were treated for a dermal sinus tract without the presence of an intraspinal dermoid cyst. Of these 113 children, $96(85.0 \%)$ had been discharged on POD 2. Seventeen patients $(15.0 \%)$ remained in the hospital for more than 2 days postoperatively. None of the 113 patients was diagnosed with a CSF leak or required return to the operating room prior to discharge.

Among the 96 patients who had been discharged on POD 2 (Table 2), 76 (79.2\%) had an isolated tethered spinal cord release and required only routine neurosurgical management during the 2-week follow-up period. Eleven patients $(11 / 96,11.5 \%)$ underwent an additional procedure under the same anesthetic as the tethered cord release, and required only routine postoperative neurosurgical care. Six patients $(6 / 96,6.3 \%)$ required nonroutine, nonoperative wound care (e.g., wound oversewing or emergency room evaluation). Three patients $(3 / 96,3.1 \%)$ required operative repair of a CSF leak or pseudomeningocele after discharge, on PODs 4, 7, and 8. Five patients in this group $(5 / 96,5.2 \%)$ were lost to follow-up after discharge.

\section{Patients Discharged After POD 2}

Seventeen patients $(17 / 113,15.0 \%)$ required hospitalization for longer than 2 days after surgery (Table 3). None had a wound-related complication identified on POD 1 or 2. The most common reasons for prolonged inpatient stays included constipation $(\mathrm{n}=5)$ and urinary retention $(n=2)$. Thirteen of the prolonged hospitalization group $(13 / 17,76.5 \%)$ required only routine neurosurgical management during the follow-up period, and 1 (5.9\%) was lost to follow-up. Two patients $(2 / 17,11.8 \%)$, following discharge on PODs 4 and 5, returned with a CSF leak and required operative repair, both on POD 8. One of these was 8 years old at the time of surgery. She presented with urinary incontinence, which also contributed to her prolonged admission. A CSF leak through a suture hole was identified at reoperation. The other patient was a 13-yearold with cerebral palsy and chronic constipation. An unintended lateral durotomy occurred, and intraoperative repair proved to be unsuccessful. Constipation was the primary reason for prolonged admission. One patient (1/17,
$5.9 \%$ ), who had been discharged to home on POD 4, was readmitted on POD 9 with a superficial wound infection, requiring operative debridement.

\section{Patients Who Underwent Coincident Procedures}

Fifteen patients $(15 / 113,13.3 \%)$ had an additional procedure performed under the same anesthetic as the simple tethered cord release. Coincident procedures are detailed in Table 4. The most common of these included exploration/excision of a potential dermal sinus tract $(n=5)$ and excision of a skin tag $(n=2)$. Four of these patients $(26.7 \%)$ had admissions longer than 2 days, and none presented with a CSF leak or infection. Two of these $(2 / 15,13.3 \%)$ were lost to follow-up after discharge.

\section{Nonroutine Postoperative Management}

Among the 107 patients assessed, 12 (11.2\%) required nonroutine neurosurgical management (operative and nonoperative) during the 14 days following surgery. This number included 5 patients $(4.7 \%)$ who required reoperation for repair of a CSF leak, $1(0.9 \%)$ who required reoperation for a superficial wound infection following exploration of a coccygeal fibrous tract and tethered cord release (Table 5), and 6 (5.6\%) who required nonoperative evaluation and management (not shown). None of these patients presented with a CSF leak or other evidence of wound complication on POD 1 or 2 . The 5 patients (4.7\%) who required reoperation for a CSF leak or pseudomeningocele had all been discharged home on or after POD 2

TABLE 2. Duration of stay

\begin{tabular}{|c|c|c|}
\hline \multirow[b]{2}{*}{ Discharge } & \multicolumn{2}{|c|}{ No. (\%) } \\
\hline & $\begin{array}{c}\text { Preintervention, } \\
n=113\end{array}$ & $\begin{array}{c}\text { Postintervention, } \\
\qquad n=56\end{array}$ \\
\hline POD 1 & $1(0.9)$ & $45(80.4)$ \\
\hline POD 2 & 95 (84.1) & $9(16.1)$ \\
\hline POD 3 & $5(4.4)$ & $1(1.8)$ \\
\hline POD 4 & $8(7.1)$ & $1(1.8)$ \\
\hline POD 5 & $1(0.9)$ & $0(0)$ \\
\hline POD 6 & $1(0.9)$ & $0(0)$ \\
\hline POD 7 & $2(1.8)$ & $0(0)$ \\
\hline Mean length of stay in days* & 2.3 & 1.3 \\
\hline
\end{tabular}


TABLE 3. Reasons for delayed discharge

\begin{tabular}{lcc}
\hline \multicolumn{1}{c}{ Reason for Delay } & \multicolumn{2}{c}{ No. $(\%)$} \\
\cline { 2 - 3 } & Preintervention; Discharge After POD 2, $\mathrm{n}=113$ & Postintervention; Discharge After POD 1, $\mathrm{n}=56$ \\
\hline Constipation & $5(4.4)$ & $1(1.8)$ \\
\hline Urinary retention & $2(1.8)$ & $0(0)$ \\
\hline Complication due to concurrent medical condition* $^{*}$ & $2(1.8)$ & $0(0)$ \\
\hline Complication of additional procedure† & $1(0.9)$ & $0(0)$ \\
\hline Severe positional headache & $1(0.9)$ & $0(0)$ \\
\hline Fever & $1(0.9)$ & $1(1.8)$ \\
\hline Pain management & $1(0.9)$ & $3(5.4)$ \\
\hline Stable postop courseł & $4(3.5)$ & $6(10.7)$ \\
\hline Wound-related complication & $0(0)$ & $0(0)$ \\
\hline Total§ & $17(15.0)$ & $11(19.6)$ \\
\hline
\end{tabular}

* Pyruvate dehydrogenase deficiency resulting in seizures and nephrolithiasis, and VACTERL syndrome (abnormalities of the vertebrae, anus, cardiovascular tree, trachea, esophagus, renal system, and limb buds) necessitating diuresis and oxygen supplementation.

$\uparrow$ Postobstructive pulmonary edema secondary to tonsillectomy.

$\ddagger$ Includes 4 patients who had evening surgery time and 2 with unclear reasons for discharge after POD 1.

$\S p=0.45$.

and returned to neurosurgical attention due to signs or symptoms related to a CSF leak. They were returned to the operating room for repair of a CSF leak on PODs 4 (1 patient), 7 (1 patient), and 8 ( 3 patients) (mean $=$ POD 7$)$.

Among the 6 patients (5.6\%) who required nonroutine, nonoperative neurosurgical care during the 2 weeks following surgery, 1 was admitted on POD 3 and underwent successful oversewing of the surgical wound; 1 was admitted on POD 4 for observation of a pseudomeningocele;

TABLE 4. Procedures performed under the same anesthetic as the tethered cord release

\begin{tabular}{|c|c|c|}
\hline \multirow[b]{2}{*}{ Procedure } & \multicolumn{2}{|c|}{ No. $(\%)$} \\
\hline & $\begin{array}{l}\text { Preintervention, } \\
\quad n=113\end{array}$ & $\begin{array}{c}\text { Postintervention, } \\
\qquad n=56\end{array}$ \\
\hline Exploration of dermal sinus tract & $5(4.4)$ & $3(5.4)$ \\
\hline Excision of skin tag & $2(1.8)$ & $2(3.6)^{*}$ \\
\hline Genitourinary surgery† & $2(1.8)$ & $2(3.6)^{*}$ \\
\hline Diagnostic proceduref & $2(1.8)$ & $0(0)$ \\
\hline Tonsillectomy/adenoidectomy & $1(0.9)$ & $0(0)$ \\
\hline Anal dilation and disimpaction & $1(0.9)$ & $0(0)$ \\
\hline $\begin{array}{l}\text { Risser cast removal and TLSO } \\
\text { mold application }\end{array}$ & $1(0.9)$ & $0(0)$ \\
\hline Myringotomy & $1(0.9)$ & $0(0)$ \\
\hline $\begin{array}{l}\text { Percutaneous Achilles tendon } \\
\text { lengthening }\end{array}$ & $0(0)$ & $1(1.8)$ \\
\hline Total§ & $15(13.3)$ & $7(12.5)^{*}$ \\
\hline \multicolumn{3}{|c|}{$\begin{array}{l}\text { TLSO = thoracolumbar spinal orthosis. } \\
\text { * One patient in the postintervention group had excision of skin tag and } \\
\text { genitourinary surgery. } \\
\text { † Included hypospadias repair, circumcision, penile reconstruction, orchio- } \\
\text { pexy, hernia repair, and anorectoplasty with transposition of anterior perineal } \\
\text { fistula. } \\
\text { † Bronchoscopy and echocardiogram. } \\
\S p=0.89 \text {. }\end{array}$} \\
\hline
\end{tabular}

2 required nonoperative care for superficial wound infections (on PODs 7 and 14); and 2 patients were evaluated for pseudomeningocele in the outpatient setting (on PODs 3 and 5) and returned home.

\section{Postintervention Group}

There were 28 girls $(50.0 \%)$ and the median age at surgery was 2.1 years. Thirty-eight patients $(67.9 \%)$ were treated for a fatty infiltrated filum terminale (with or with-

\section{TABLE 5. Patients requiring reoperation}

\begin{tabular}{lll}
\hline & \multicolumn{2}{c}{ No. (\%) } \\
\cline { 2 - 3 } Patient Characteristic & $\begin{array}{c}\text { Preintervention, } \\
\mathrm{n}=107\end{array}$ & $\begin{array}{c}\text { Postintervention, } \\
\mathrm{n}=53\end{array}$ \\
\hline $\begin{array}{c}\text { Total patients requiring operative } \\
\text { intervention }\end{array}$ & $6(5.6)$ & $1(1.9)$ \\
\hline Diagnosis & \\
\hline Fatty filum terminale & $4(3.7)$ & $0(0)$ \\
\hline $\begin{array}{l}\text { Low-lying conus w/o fatty } \\
\text { filum }\end{array}$ & $2(1.9)$ & $1(1.9)$ \\
\hline Dermal sinus tract & $0(0)$ & $0(0)$ \\
\hline POD of discharge & & $0(0)$ \\
\hline 1 & $0(0)$ & $1(1.9)$ \\
\hline 2 & $3(2.8)$ & $0(0)$ \\
\hline$>2 *$ & $3(2.8)$ & $1(1.9)$ \\
\hline POD of re-presentation & & $0(0)$ \\
\hline$\leq 7$ & $2(1.9)$ & \\
\hline $8-14$ & $4(3.7)$ & $1(1.9)$ \\
\hline Indication for reop & & $0(0)$ \\
\hline CSF leak & $5(4.7)$ & $0(0)$ \\
\hline Superficial wound infection & $1(0.9)$ & \\
\hline Coincident procedure & $0(0)$ & \\
\hline * Discharge was on POD 4 or 5. & & \\
\hline
\end{tabular}


TABLE 6. Length of admission-breakdown by surgeon*

\begin{tabular}{|c|c|c|c|c|c|c|c|c|c|c|c|c|}
\hline \multirow{2}{*}{$\begin{array}{l}\text { Admission } \\
\text { Category† }\end{array}$} & \multicolumn{3}{|c|}{ Surgeon A } & \multicolumn{3}{|c|}{ Surgeon B } & \multicolumn{3}{|c|}{ Surgeon C } & \multicolumn{3}{|c|}{ Surgeon D } \\
\hline & Pre & Post & Total & Pre & Post & Total & Pre & Post & Total & Pre & Post & Total \\
\hline No. w/ planned (\%) & $31(96.9)$ & $22(95.7)$ & $53(96.4)$ & $27(84.4)$ & $9(69.2)$ & $36(80.0)$ & $21(87.5)$ & $11(73.3)$ & 32 (82.1) & $17(68.0)$ & $3(60.0)$ & $20(66.7)$ \\
\hline $\begin{array}{l}\text { No. w/ prolonged } \\
(\%)\end{array}$ & $1(3.1)$ & $1(4.3)$ & $2(3.6)$ & $5(15.6)$ & $4(30.8)$ & $9(20.0)$ & $3(12.5)$ & $4(26.7)$ & $7(17.9)$ & $8(32.0)$ & $2(40.0)$ & $10(33.3)$ \\
\hline Total & 32 & 23 & 55 & 32 & 13 & 45 & 24 & 15 & 39 & 25 & 5 & 30 \\
\hline
\end{tabular}

out a low-lying conus medullaris); 17 (30.4\%) were treated for a low-lying conus medullaris in the absence of fat in the filum terminale; and $1(1.8 \%)$ was treated for a dermal sinus tract without the presence of an intraspinal dermoid cyst (Table 1). Of these 56 children, 45 (80.4\%) were discharged on POD 1. Eleven patients (19.6\%) remained in the hospital for more than 1 day postoperatively (Table 2).

Among the 42 patients who were discharged on POD 1 and had documented follow-up, 35 (83.3\%) had both an isolated tethered spinal cord release and required only routine neurosurgical management during the 2 -week follow-up period. Among the 56 total patients, 7 (12.5\%) underwent an additional procedure under the same anesthetic as the tethered cord release, and required only routine postoperative neurosurgical care. No patient required nonroutine, nonoperative wound care. Among the 53 patients with follow-up, $1(1.9 \%)$ required operative repair of a CSF leak, on POD 4. Three patients $(3 / 56,5.4 \%)$ were lost to follow-up after discharge.

\section{Patients Discharged After POD 1}

Eleven patients $(11 / 56,19.6 \%)$ required hospitalization for longer than 1 day after surgery (Table 2). None had a wound-related complication identified on POD 1 or 2. The most common reasons for prolonged inpatient stays included evening surgery time $(n=4)$ and inadequate postoperative pain control $(n=3)$. There were 2 patients for whom record review did not reveal a clear reason for discharge after POD 1 (Table 3). The patient who required return to the OR for a CSF leak had been discharged on POD 2 due to a late surgery time.

\section{Patients Who Underwent Coincident Procedures}

Six patients $(6 / 56,10.7 \%)$ had an additional procedure performed under the same anesthetic as the simple tethered cord release. Coincident procedures are detailed in Table 4. The most common of these included exploration and/or excision of a potential dermal sinus tract $(n=3)$. One of these 6 patients $(16.7 \%)$ had an admission longer than 1 day, and none presented with a CSF leak or infection. None were lost to follow-up after discharge.

\section{Nonroutine Postoperative Management}

Among the 53 patients assessed, 3 (5.7\%) required nonroutine neurosurgical management during the 14 days fol- lowing surgery (Table 5). This included 1 patient $(1.9 \%)$ who required reoperation for repair of a CSF leak (POD 4), $1(1.9 \%)$ who required readmission for oversewing of a CSF leak (POD 7), and 1 who required admission and observation of a pseudomeningocele without intervention (POD 3). The patient who required repair of a CSF leak was a 16-year-old with Down syndrome who, at reoperation, was noted to have leaking through the dural closure.

\section{Analysis by Surgeon}

Four surgeons performed operations that were included in the analysis. Statistical analysis of CSF leak rates did not demonstrate an association with surgeon (rates ranged from $1.8 \%$ to $5.5 \%$ ). There was an association between the likelihood of on-time discharge and the surgeon who provided treatment (Table 6). This resulted from the difference between 1 surgeon, whose patients were less likely to have a prolonged admission, and the other 3 surgeons.

\section{Discussion}

There are currently no published guidelines for the perioperative management of children who have undergone a simple tethered spinal cord release. Until recently, the institutional practice at Children's Hospital Colorado had been to maintain children in a horizontal position for 24 hours after surgery, to liberalize activities on POD 1, and to monitor the incision site for evidence of a pseudomeningocele or CSF leak until POD 2. This practice was modified to allow for the discharge of patients on the afternoon of POD 1. Our experience before and after this change demonstrates no increased risk of a perioperative CSF-related complication following simple tethered spinal cord release (defined as those without a substantial fascial or dural defect related to the tethering lesion). Therefore, in the absence of other reasons for the patient to remain in the hospital, we have maintained the new practice of keeping patients lying flat overnight after surgery, and discharging them on the afternoon of POD 1.

Although this quality improvement initiative did not compare strategies regarding the length of time that patients were kept flat, our finding of a 3.8\% (6/160) postoperative CSF leak rate was between the $5.9 \%$ rate reported by Chern and colleagues ${ }^{1}$ and the $1.9 \%(1 / 52)$ and $0 \%$ rates reported by Cools and colleagues ${ }^{3}$ and Ogiwara and colleagues, respectively. ${ }^{7}$ Both Chern and Ogiwara found that 
the duration of postoperative recumbency, whether in the hospital or at home, was not associated with the development of a CSF leak following release of a tight filum terminale. As opposed to the least restrictive strategy in either of these studies, ${ }^{1}$ which included 24 hours of recumbency in the hospital followed by 48 hours of in-home recumbency, we allowed full activities after approximately 24 hours, followed by a brief period of inpatient monitoring.

As a quality improvement initiative using existing data from a single center, our findings may not be generalizable. In addition, the retrospective data used to complete this project do not offer adequate detail to determine whether subtle clinical features could have warned of impending complications. For example we could not detect if, on POD 1 , a member of the clinical team had a suspicion that a patient who ultimately required repair of a CSF leak on POD 4 might have a small fluid collection at the incision site.

An alternative conclusion from these data could also be that patients should be kept in the hospital for a longer period of observation. In our cohort, the mean time to reoperation was 6.2 days. With the assumption that the decision to reoperate was made on POD 5 , we estimate that a protocol intended to keep children under inpatient observation until this point would require approximately 106.7 hospital days for each leak detected, when compared with discharge on POD 1.

\section{Conclusions}

Through a single-center quality improvement initiative, we found that shortening the period of inpatient monitoring following laminectomy or laminotomy for release of a simple tethered spinal cord was not associated with an increased likelihood of CSF-related complications at Children's Hospital Colorado. Although this project did not provide evidence for an optimal duration of postoperative recumbency, the $3.8 \%$ rate of reoperation for CSF-related complications is consistent with previous publications that showed no advantage associated with longer periods of horizontal positioning.

\section{Acknowledgments}

Dr. Hankinson received funding from the Colorado Clinical and Translational Sciences Institute/Children's Hospital Colorado Research Institute (KL2 Research Scholar Award [NCATS/NIH UL1 TR001082]), and from the Morgan Adams Foundation.

\section{References}

1. Chern JJ, Tubbs RS, Patel AJ, Gordon AS, Bandt SK, Smyth $\mathrm{MD}$, et al: Preventing cerebrospinal fluid leak following transection of a tight filum terminale. J Neurosurg Pediatr 8:35-38, 2011

2. Cohrane DD: Occult spinal dysraphism, in Albright AL, Pollack IF, Adelson PD (eds): Principles and Practice of Pediatric Neurosurgery, ed 3. New York: Thieme, 2014

3. Cools MJ, Al-Holou WN, Stetler WR Jr, Wilson TJ, Muraszko KM, Ibrahim M, et al: Filum terminale lipomas: imaging prevalence, natural history, and conus position. J Neurosurg Pediatr 13:559-567, 2014

4. Guerra LA, Pike J, Milks J, Barrowman N, Leonard M: Outcome in patients who underwent tethered cord release for occult spinal dysraphism. J Urol 176:1729-1732, 2006

5. Koyanagi I, Iwasaki Y, Hida K, Abe H, Isu T, Akino M: Surgical treatment supposed natural history of the tethered cord with occult spinal dysraphism. Childs Nerv Syst 13:268274, 1997

6. Kulkarni AV, Pierre-Kahn A, Zerah M: Conservative management of asymptomatic spinal lipomas of the conus. Neurosurgery 54:868-875, 2004

7. Ogiwara H, Joko M, Takado M, Uematsu K, Kameda M, Sasaki N, et al: Duration of the horizontal decubitus position for prevention of cerebrospinal fluid leakage following transection of a tight filum terminale. J Neurosurg Pediatr 15:461-464, 2015

8. Ogiwara H, Lyszczarz A, Alden TD, Bowman RM, McLone DG, Tomita T: Retethering of transected fatty filum terminales. J Neurosurg Pediatr 7:42-46, 2011

9. Pang D, Zovickian J, Oviedo A: Long-term outcome of total and near-total resection of spinal cord lipomas and radical reconstruction of the neural placode, part II: outcome analysis and preoperative profiling. Neurosurgery 66:253273,2010

\section{Disclosures}

The authors report no conflict of interest concerning the materials or methods used in this study or the findings specified in this paper.

\section{Author Contributions}

Conception and design: Hankinson, Wilkinson, O'neill, Handler. Acquisition of data: all authors. Analysis and interpretation of data: Hankinson, Poonia, Graber. Drafting the article: Hankinson, Poonia. Critically revising the article: Hankinson, Poonia, Wilkinson, O'neill, Handler. Reviewed submitted version of manuscript: all authors. Approved the final version of the manuscript on behalf of all authors: Hankinson. Statistical analysis: Graber.

\section{Correspondence}

Todd C. Hankinson, Pediatric Neurosurgery, Children's Hospital Colorado, University of Colorado Anschutz Medical Campus, 13123 E. 16th Ave., Box 330, Aurora, CO 80045. email: todd. hankinson@childrenscolorado.org. 